Guillermo Tortolero-Luna 1 Michele Follen Mitchell 1 David C. Swan 2 Ruth Ann Tucker 2 LouiseWideroff 3 Joseph P. Icenogle 2

\section{A case-control study of human papillomavirus and cervical squamous intraepithelial lesions (SIL) in Harris County, Texas: differences among racial/ethnic groups}

\author{
Um estudo de caso-controle de infecção por \\ papillomavirus humano e câncer de colo uterino \\ em Harrys County, Texas
}

1 Department of Gynecologic Oncology University of Texas M.D. Anderson Cancer Center, Houston, Texas 77030-4095,USA.

2 National Center for Infectious Diseases, Centers for Disease Control and Prevention. Mail Stop G-18, 1600 Clifton Rd., Atlanta,GA,30333,U SA. 3 Applied Research Branch, Division of Cancer Control and Population Science, National Cancer Institute. 6130 Executive Bl vd. ,M SC 7344, Bethesda, Maryland 20892-7344,USA.
Abstract Weconducted a case-control study of the association between SIL and HPV among whites (W), African Americans (AA), and Hispanics (H) in Harris County, Texas. Cases were identified at M.D. Anderson Cancer Center Colposcopy Clinic. Controls were identified among women obtaining routine Pap screening at two Harris County Health Department Clinics. HPV was detected by a PCR-based fluorescent assay. Dichotomous and polytomous logi stic regression model s were used to estimate adjusted odd ratios (AOR) and 95\% confidence intervals $(\mathrm{Cl})$ for SI L among racial/ethnic groups and grade of disease. Prevalence of HPV infection was $64 \%$ in low grade SIL (LSIL), 84\% in high grade SIL (HSIL), and 19\% in controls. Ri sk of SIL was higher in $\mathrm{H}$ than in $\mathrm{W}$ and $A A, A O R 29.5$ (12.4-70.5), 15.3 (6.0-33.8), and 5.8 (2.6- 12.6), respectively. Similarly, racial/ethnic differences were observed for both LSIL (AOR $=16.6,7.7$, and 4.3, respectively) and HSIL (AOR =78.6, 34.6, and 14.2, respectively). Findings support the association between SIL and HPV and differences in the strength of the association with LSILs and HSILs. Data also suggest a higher risk for $\mathrm{H}$ and a lower risk for $\mathrm{AA}$.

Key words Papillomavirus; Cervix Neoplasms; Ethnic Groups; Case-Control Studies

Resumo Foi realizado um estudo caso-controle para analisar a associação entre lesões intraepitel iai s escamosas do colo uteri no (SI L) e HPV entre mulheres brancas, negras elatinas em Harris County, Texas. Os casos foram identificados na M. D. Anderson Cancer Center Col poscopy Clinic, e os controles foram obtidos realizando-se exame de Papanicolau em duas clínicas do Departamento de Saúde. O HPV foi detectado por mei o de ensaio de PCR (primer MY09/MY11). Foram construí dos model os de regressão logística para esti mar as odds ratios ajustadas (AOR), e seus interval os de confiança de $95 \%$ (IC) de SI L entre os grupos étnicos e graus da doença. A pre valência de HPV nas SIL de baixo grau (LSIL) foi de 64\%; nas de alto grau (HSIL), 84\%; e 19\% nos controles. O risco de SIL foi maior em mulheres latinas que em brancas e negras, sendo observadas, respectivamente, as seguintes AOR: 29,5 (12,4-70,5); 15,3 (6,0-33,8); e 5,8 (2,6-12,6). De forma similar, foram observadas diferenças para ambos LSIL (AOR, respectivamente, de 16,6; 7,7 e 4,3) e HSIL (AOR de 78,6; 34,6 e 142). Os resultados apóiam a existência de associação entre SI L e HPV, diferenças na força de associação com SI Lse HSILs, e sugerem risco mais el evado para muIheres latinas e menor para mulheres negras.

Palavras-chave Papilomavirus; Neoplasias do Colo Uterino; Grupos Étnicos; Estudos de Casose Controles 
Introduction

During the last decade, several epidemiological and experimental studies have documented a strong association between cervical neoplasia and human papillomavirus (HPV) infection (Muñoz, 1997). The association has been observed for both invasive and pre-invasive cervical neoplasias, and the results have been consistent worldwide (Bosch et al., 1993; 1995; Shiffman et al., 1993; Becker et al., 1994; Liaw et al., 1995; Olsen et al., 1995). Although incidence and mortality from cervical cancer in the U.S. have declined during the last 50 years, racial/ ethnic differences remain. Women from underrepresented racial/ ethnic groups and low-income women continue to carry a higher burden from this neoplasm. In the U.S., incidence rates are higher among Vietnamese, Hispanic, African American, Native Alaskan, and Korean women (Becker et al., 1992; Miller et al, 1996). Similar disparities in incidence and mortality rates from cervical cancer are reported in Texas. Incidence rates in Texas are higher in Hispanics, intermediate in African American, and lower in whites.

Despite this, few studies have examined the role of racial/ethnic differences in risk factors associated with cervical neoplasia. It is necessary to determine if differences in the prevalence of these factors and their association with the cervical neoplasia might help explain the racial/ ethnic disparities in incidence and mortality rates (Napoles-Springer et al., 1996). Little is known about differences in the prevalence of biological factors such as HPV infection and of HPV types among racial/ethnic groups and their association with cervical neoplasia. Becker et al. (1994) reported differences between Hispanic and non-Hispanic white women in New Mexico in the risk of HSI L associated with HPV infection. A stronger association between HPV and high-grade dysplasia, particularly among HPV 16 and 18 positive women, was observed for Hispanics. In addition, Bosch et al. (1993; 1995) have shown geographic differences in the prevalence of HPV infection and HPV types.

We conducted a multi-ethnic, clinic based, case-control study to assess the association between squamous intraepithelial lesions (SI Ls) and HPV DNA status and other risk factors among women from three racial/ ethnic groups (white, African American, and Hispanic) residing in Harris County, Texas. In this report, we focus on the role of HPV status among white, African-American, and Hispanic women in the risk of SIL (low-grade SIL [LSIL] and high-grade SIL [HSIL]).
Material and methods

\section{Study population}

This study was conducted in Harris County, TX between September 1991 and August 1994. Eligible cases were identified among women referred to the University of Texas M. D. Anderson Cancer Center (UTMDACC) Colposcopy Clinic for further evaluation of an abnormal Pap smear. This clinic provides colposcopy services for minority and economically disadvantaged women with abnormal Pap smears referred from Health Department clinics in Harris County. Only women with a confirmed histological diagnosis of squamous intraepithelial lesion (SIL) were included as cases. Controls were selected among women attending family planning and screening services at two Harris County Health Department (HCHD) clinics. The selection of the two HCHD clinics for recruitment of control subjects was based on the large and multi-ethnic population to which they provide medical care. Women eligible for the control group were included in the study if the cytological smear at the time of recruitment was reported as normal. Both cases and controls were non-pregnant white, AfricanAmerican, or Hispanic women aged 18 years and older, and residents of Harris County at the time of the study. Other eligibility criteria included no previous history of cervical neoplasia and/or treatment for cervical neoplasia, cancer, or hysterectomy. In addition, women in the control group had a negative history of abnormal Pap smear or cervical biopsy.

\section{Data collection}

Women were screened for eligibility prior to receiving medical care. Eligible women were asked to participate in the study, sign an informed consent, complete a two-part interview, and provide a $20 \mathrm{cc}$ blood sample. Interviews and specimen collection were obtained during the first visit to the clinic and prior to final cytological or histological diagnosis. In order to insure the participation of Spanish speaking women, interviews were offered in both English and Spanish. The first part of the interview included detailed information on: demographic characteristics (age, race/ ethnic background, marital status, education, income, and religious preference); sexual behavior (number of sexual partners, age at first sexual intercourse; and sexual practices); reproductive history (age at menarche, age at first birth and parity, and date of last menstrual pe- 
riod); smoking history (smoking status, duration, and intensity of smoking habits); contraceptive history (history or current use of contraceptive methods, type of contraceptive method used, and duration of use); Pap smear frequency; and medical history (sexually transmitted diseases and immunosuppressive disorders). The second part of the interview consisted of a computer-assisted food frequency questionnaire using the "Health Habits and History Questionnai re: Diet History and Other Risk Factors" (Block, 1989). Women were asked their average frequency of consumption of 98 food items during the year prior to enrollment.

Cases had a complete physical and pelvic examination, repeat Pap smear, colposcopic examination, colposcopically directed biopsies of abnormal areas, collection of two cervical samples for HPV testing by ViraPap/ViraType ${ }^{\text {mm }}$ (standard care at the UTM DCC) and polymerase chain reaction (PCR). The medical staff of the colposcopy clinic (a gynecologic oncologist, two gynecologists, two family physicians, and two colposcopy-trained nurse practitioners) conducted examinations and specimen collection. Women in the control group al so had a complete physical and pelvic examination, Pap smear, and collection of two cervical samples for HPV testing by ViraPap/ViraType ${ }^{\mathrm{Tm}}$ and PCR. Exams and specimen collection were performed by two nurse practitioners trained at the UTMDACC. Samples for ViraPap/ViraType $^{\mathrm{m} m}$ testing were collected with a cotton swap and preserved in the media transport provided by the manufacture. The samples for PCR analysis were collected with cervical brushes. Cervical brushes were stored in vials and refrigerated immediately in dry ice and later at $-70 \circ \mathrm{C}$ until HPV testing by PCR was conducted.

In addition, a 20cc blood sample was obtained from all study participants for serum determinations of retinol and $\beta$-carotene and other serologic studies. Blood samples were collected in EDTA vacutainer tubes, protected from exposure to light with aluminum foil, and stored immediately in dry ice. Within 4 hours after venipuncture, the samples were centrifuged and serum was stored in $1 \mathrm{ml}$ aliquots at $-70 \circ \mathrm{C}$ until analysis.

Cytological and histological diagnosis

Cytological and histological specimens for all cases were interpreted at the UTM DACC Department of Pathology. Two independent readers at the UTM DACC reviewed each Pap smear and biopsy. A committee of staff members including the Director of the Colposcopy Clinic
(MFM) reviewed discrepant cases monthly and reached a final diagnosis. Cytological specimens for the control group followed the normal process at the Harris County Health Department. All specimens were read and interpreted at a centralized laboratory at the San Antonio Chest Hospital, in San Antonio, Texas. A copy of the cytology report was obtained from the HCHD clinics for all patients who had completed an interview in order to determine final eligibility.

Overall, 1,776 women were screened for eligibility ( 1,264 at UTMDACC and 512 at HCHD clinics). Six hundred and twenty-six patients (36\%) were non-eligible because of residency out of Harris County. Additionally, 329 were non-eligible because of: prior history and treatments of cervical abnormality ( $n=105)$; pregnancy $(n=104)$; age less than 18 years $(n=60)$; Hysterectomy $(n=13)$, and other reasons $(n=$ 40). At UTMDACC 659 women who met eligibility at the time of the first visit accepted to participate. Sixty four percent (399 women) meet the eligibility criteria of histological diagnosis of SIL. After excluding 45 additional cases for lack of HPV data and 29 for incomplete data, the case group included 325 women. At the HCHD clinics, 328 women were el igible and accepted to participate. Twenty-six women were later excluded because they did not meet cytological criteria (negative cytology) for inclusion and 32 were excluded because of the lack of HPV data. Therefore, 270 controls were available for the study.

HPV testing

The laboratory methods have been previously described (Swan et al., 1997). DNA specimens were obtained from cytobrush samples. Cytobrush samples were thawed and vortexed in 1 $\mathrm{ml}$ 0.01M PBS, 5 mM EDTA, pH 7.4. Samples were centrifuged at $1,000 \times \mathrm{g}$ for $5 \mathrm{~min}$ at room temperature. Supernatants were removed and saved. DNA was extracted from each pellet by suspension in $200 \mu \mathrm{l} 10 \mathrm{mM}$ Tris, $\mathrm{pH} 7.5,1 \mathrm{mM}$ EDTA, $0.5 \%$ SDS containing $100 \mu \mathrm{g} / \mathrm{ml}$ Proteinase $K$, incubation at 650 for one hour, transfer to Phase-Lock gel tubes (5 Prime $\rightarrow 3$ Prime, Boulder, CO 80303) containing $200 \mu \mathrm{l}$ phenolchloroform-isoamyl alcohol (25:24:1), mixing by inversion and phase separation at 12,000 $\mathrm{xg}$ for $2 \mathrm{~min}$. An additional $100 \mu \mathrm{l}$ chloroform was added per tube, and the tube contents were mixed and centrifuged as above. Each supernatant from the Phase-Lock procedure was diluted to $2 \mathrm{ml}$ with water and centrifuged in a Centricon 100 microconcentrator (Amicon, 
Inc., Beverly, MA) at $1000 \times \mathrm{g}$ for $30 \mathrm{~min}$. An additional $2 \mathrm{ml}$ of water was added to each retentate, and the centrifugation was repeated. Retentates were then collected and diluted to 200 $\mu \mathrm{l}$ with water. $10 \mathrm{ul}$ of this DNA was used in each fluorogenic $P C R$ reaction. A contamination control for $P C R$ reactions consisting of 1 $\mathrm{ml}$ water was inserted after every tenth patient sample and subjected to the entire extraction and DNA detection protocol. Each DNA specimen was tested for overall HPV positivity by PCR using consensus primers followed by electrophoresis in ethidium bromide-containing gels (Manos et al., 1989). HPV-positive samples were tested by the quantitative fluorescent probe assay for HPV types 16, 18, 31, and 45. This paper focuses only on the results from the consensus primer.

Probes: The fluorigenic probe assay is based on the increase in fluorescent signal which occurs when probes are degraded by the $5^{\prime} \rightarrow 3^{\prime}$ exonuclease activity of Taq polymerase (Förster, 1948). After degradation, the reporter dye ((FAM)6-carboxyfluorescein or (HEX)hexachloro-fluorescein), present at the 5 '-end of each probe can diffuse away from a quencher dye ((TAM RA)6-carboxy-tretramethyl-shodamine) present on or near the 3'-end of each probe thereby increasing the fluorescent signal from the reporter dye. The probe sequences for each of the high-risk HPVs were selected and synthesized as described previously (Swan et al., 1997).

Primers: The primer sequences were selected using the Oligo 5.0 primer analysis program (National Biosciences, Inc., Plymouth, MN). The primer pairs for each of the HPV types were selected based on having a Tm of approximately 65o, predicted lack of cross-hybridization to other common HPV types, no predicted loop formation and no predicted dimer formation with the other primer.

Control templates: Control templates for HPV types 16, 18, 31, 33, 35, 45, 51, 52 and 56 were prepared by PCR amplification of cloned DNA using L1, type-specific primers (sequences available on request) and purified using Centricon 100 microconcentrators. The DNA concentrations were determined by fluorometry (DyNA Quant 200, Hoefer-Pharmacia Biotech, San Francisco, CA) using $2 \mu$ of DNA solution in $2 \mathrm{ml}$ freshly prepared Hoechst 33258 dye solution $(1 \mu \mathrm{g} / \mathrm{ml}$ in $10 \mathrm{mM}$ Tris $\mathrm{pH}$ 7.4, 1 mM EDTA, $200 \mathrm{mM} \mathrm{NaCl}$ ) (Pharmacia Biotech Inc., Piscataway, NJ). A $100 \mu \mathrm{g} / \mathrm{mI}$ DNA standard was used.

Assay controls consisting of a dilution series of the homologous template ( $1 \times 105$ to $3 \times 101$ copies) and a set of heterologous templates (2x103 copies of HPV types 16, 18, 31, 33, 35, 45, 51,52 and 56 in separate tubes) were included in each run. Each control sample also contained 50 ng human placental DNA.

Fluorigenic PCR. The $50 \mu \mathrm{l}$ PCR mixtures contained $10 \mathrm{mM}$ Tris $\mathrm{pH} 8.3,50 \mathrm{mM} \mathrm{KCl}, 4.5$ $\mathrm{mM} \mathrm{MgCl}_{2}, 200 \mu \mathrm{M}$ dNTP's, $0.3 \mu \mathrm{M}$ each primer, $50 \mathrm{nM}$ each fluorigenic probe (FAMHPV probe and HEX-globin probe), $0.025 \mathrm{U} / \mu \mathrm{l}$ Taq Gold polymerase (Perkin-Elmer Corp., Norwalk, CT) and $10 \mu$ template DNA. Following Taq polymerase activation and template denaturation for $12 \mathrm{~min}$ at 950, amplification conditions were as follows: 40 cycles of $30 \mathrm{sec}$ at $940,10 \mathrm{sec}$ at $60^{\circ}$ and $2 \mathrm{~min}$ at $65^{\circ}$. Fluorescence measured in a Perkin-Elmer LS-50B luminescence spectrometer. Data acquisition and analysis were performed using the TaqMan Fluorescence Data Manager (Perkin-Elmer Corp.) and Excel 5.0 (Microsoft Corporation, Redmond, WA).

Copy Number Calculation: The spill-over fluorescence from the FAM (HPV) channel into the HEX (globin) channel and vice versa was calculated from two sets of control samples; the first was a set of three identical samples containing both probes but only HPV template and the second was a set of three containing both probes but only globin template. HPV and globin signals were calculated in patient samples after subtraction of the spill-over signal. Included with each set of patient samples were the assay controls and a dilution series of nonHPV-containing human cellular DNA. Plots of the homologous template dilution series fluorescence versus log (template copies) were linear over the range 50 to $1 \times 109$ copies; hence from the patient fluorescence data it was possible to calculate HPV copy number in patient samples. Extrapolation of log (copy number) versus fluorescence above $1 \times 109$ copies is of unknown accuracy; however, samples with fluorescence corresponding to copy numbers in this range can be presumed to have at least 109 copies. Median values of HPV copy number in the present study do not exceed 108 and thus, inaccuracies in HPV copy number above 109 will not affect conclusions based on median copy number values.

\section{Sample size and statistical analysis}

Sample size was estimated based on a conservative prevalence of HPV in the control and case groups of $20 \%$ and $40 \%$, respectively. Assuming a $20 \%$ prevalence of HPV infection among cytologically normal women, we estimated that a 
sample of 63 cases and controls per racial/ ethnic group would detect a minimum threefold risk difference between cases and controls with a two-sided test, alpha of .05 and $80 \%$ power. Due to the racial/ ethnic distribution of the clinic population at the time of the study (approximately $60 \%$ white, $20 \%$ African-American and $20 \%$ Hispanic) and study recruitment procedures (enrollment prior to final diagnosis) we expected to enroll a larger number of white women and had planned to randomly select one of every two or three white cases. We conducted a preliminary analysis to estimate crude odds ratios (OR) for the association between SIL combined and HPV for all racial/ethnic groups combined using the total number of available white cases ( $n=147)$ in the study sample and a random sample $(n=70)$ of the white cases as originally planned. Because no important differences were observed between both risk estimates, we present results for the entire sample.

Comparisons between cases and controls and among racial/ ethnic groups were conducted using the Pearson's X2 test for categorical variables (HPV status, income, education, number of sexual partners, etc. ) and ' $t$ ' test and oneway ANOVA for continuous variables (age and education). Odd ratios and $95 \%$ confidence intervals $(\mathrm{Cl})$ were used to estimate the association between SIL combined and LSIL and HSI L and HPV DNA status (HPV DNA positive or negative) and other known risk factors. Two types of logistic regression models were estimated: dichotomous logistic regression models for SI Ls combined and polytomous logistic regression models for grade of lesion (LSIL and HSI L) (Hosmer \& Lemeshow, 1989). This model simultaneously estimates the risk of LSI L and HSIL with respect to a common comparison group. Separate dichotomous and polytomous models were performed for the combined sample and for each racial/ethnic group.

In addition to HPV DNA status and age, variables with a $p$ value $<0.05$ in the univariate logistic regression analysis were considered for inclusion in the multivariate models. Similar procedure was followed for the analysis of the combined sample and each racial/ethnic group separately. Variables in the final models included: age (continuous), HPV DNA status (positive, negative), age at first sexual intercourse $(<17,17-19,20+$ [reference category]), lifetime number of sexual partners ( 1 [reference category], 2-3, 4-5, 6+); smoking history (ever, never), and history of STDs (yes, no).

Statistical analyses were performed using the Statistical Package for the Social Sciences
(SPSS, 1997) and STATA (Statacorp., 1997). All p values are for two-sided tests, and statistical significance was set at $p<0.05$.

\section{Results}

Five hundred ninety-five women were included in this analysis, 325 cases ( 175 LSIL and 150 $\mathrm{HSIL}$ ) and 270 controls. Among women with a diagnosis of LSIL, 84 (48\%) had koilocytotic atypia and 91 (52\%) had cervical intraepithelial neoplasia ( $\mathrm{CIN}$ ) grade 1 . Whereas, among women with a diagnosis of HSIL, 70 (47\%) had CIN 2 and 79 (53\%) had CIN 3. Thirty-six percent of all study subjects were white (147 cases and 68 controls), 25\% were African American ( 75 cases and 73 controls), and $39 \%$ were Hispanic ( 103 cases and 128 controls). Ninety-nine percent $(n=589)$ of women were $\leq 45$ years of age (mean age 26.6 years and standard deviation 6.4). The study subjects were of low socioeconomic status with $86 \%$ reporting an annual family income of less than $\$ 20,000$ and $50 \%$ having less than high school education.

Comparisons of demographic characteristics between cases and controls are presented in Table 1. No statistically significant difference between SIL cases combined and controls were observed in mean age ( $p=0.35)$, education level $(p=0.25)$, and income $(p=0.65)$. Similarly, no statistically significant differences in education and family income were observed between controls and LSI L and HSIL. However, LSIL cases were slightly younger than controls ( $p=$ 0.004).

In addition, cases and controls differed in the prevalence of several known risk factors for cervical neoplasia. Smoking history was higher among cases (47\%) than in controls $(26 \%$, p < 0.001). The case-control difference in smoking history was Iarger between HSIL (57\%) than between LSI L (37\%) cases. Approximately 91\% of cases had their first sexual intercourse before age 20 compared with $83 \%$ of controls ( $p$ $\varangle 001$ ). Cases were less likely to report a single lifetime sexual partner (15\%) and to report six or more sexual partners (35\%) than women in the control group ( $36 \%$ and $16 \%$, respectively, $\mathrm{p}<0.001$ ). Similarly, cases were more likely to have a history of STDs (33\%) than controls (17\%) ( $p<0.001$ ). No differences between cases and controls were detected for the number of pregnancies and oral contraceptive use.

Case-case comparisons (LSIL vS. HSIL) showed that compared with LSIL cases, women with a diagnosis of HSIL were slightly older (mean 27.7 vs. 25.2, p =0.002) and more likely 
Table 1

Distribution of demographic characteristic among cervical squamous intraepithelial lesions (SIL) cases and controls by grade of lesion.

\begin{tabular}{|c|c|c|c|c|c|}
\hline \multirow[t]{2}{*}{ Risk factor } & \multicolumn{2}{|c|}{ SIL } & \multirow[b]{2}{*}{$\begin{array}{c}\text { Controls } \\
\text { n (\%) }\end{array}$} & \multirow[b]{2}{*}{$\begin{array}{c}p^{1} \\
n(\%)\end{array}$} & \multirow[b]{2}{*}{$\begin{array}{c}p^{2} \\
n(\%)\end{array}$} \\
\hline & LGSIL & HGSIL & & & \\
\hline Age (years) & & & & 0.004 & 0.274 \\
\hline Mean $( \pm S D)$ & $25.3(5.9)$ & $27.7(8.1)$ & $26.9(5.6)$ & & \\
\hline Race/Ethnic group & & & & $<0.001$ & $<0.001$ \\
\hline White & $67(38)$ & $80(53)$ & $68(25)$ & & \\
\hline African-american & $53(30)$ & $22(15)$ & $74(27)$ & & \\
\hline Hispanics & $55(31)$ & $48(32)$ & $128(47)$ & & \\
\hline Education & & & & 0.060 & 0.869 \\
\hline >High School & $52(31)$ & $36(25)$ & $63(24)$ & & \\
\hline High School & $73(44)$ & $53(37)$ & $105(39)$ & & \\
\hline$<$ High School & $42(25)$ & $56(39)$ & $100(37)$ & & \\
\hline Income & & & & 0.903 & 0.731 \\
\hline$<\$ 20,000$ & $149(86)$ & $129(86)$ & $228(85)$ & & \\
\hline$\$ 20,000$ & $24(14)$ & $21(14)$ & 41 (15) & & \\
\hline Marital status & & & & $<0.001$ & 0.012 \\
\hline Single & $83(50)$ & $43(30)$ & $82(31)$ & & \\
\hline Married & $41(25)$ & $64(44)$ & $146(55)$ & & \\
\hline Divorced & $39(23)$ & $37(26)$ & $35(13)$ & & \\
\hline Widowed & $4(2)$ & $1(1)$ & $4(2)$ & & \\
\hline
\end{tabular}

$1 \mathrm{p}$ value for the comparison between LSIL vs. control group.

$2 \mathrm{p}$ value for the comparison between HSIL vs. control group.

Table 2

Prevalence of HPV Infection by case-control status, racial/ethnic group and grade of lesion.

\begin{tabular}{lcrrr}
\hline Race/Ethnic group & $\begin{array}{c}\text { SIL combined } \\
n(\%)\end{array}$ & $\begin{array}{c}\text { SIL } \\
\text { LGIL }\end{array}$ & $\begin{array}{c}\text { HGSIL } \\
n(\%)\end{array}$ & $\begin{array}{c}\text { Controls } \\
n(\%)\end{array}$ \\
\hline White & $112(76)$ & $43(64)$ & $69(86)$ & $13(19)$ \\
African-american & $51(68)$ & $34(64)$ & $17(77)$ & $22(30)$ \\
Hispanic & $75(73)$ & $35(64)$ & $40(83)$ & $17(13)$ \\
All & $238(73)$ & $112(64)$ & $126(84)$ & $52(19)$ \\
P value1 & NS & NS & NS & 0.017
\end{tabular}

$1 \mathrm{p}$ value for the difference between racial/ethnic groups in each group.

to be white than Hispanic or African American (54\%, 47\%, and $29 \%$, respectively). Women with $\mathrm{HSIL}$ were more likely to be to less educated than women with LSI L (<high school education $39 \%$ and $25 \%$, respectively), to have a history of smoking ( $57 \%$ vs. $39 \%$ ), and to have had four or more pregnancies ( $23 \%$ vs. $12 \%$, $p=0.026)$. However, LSIL and HSIL cases did not differ in age at first intercourse, number of lifetime sexual partners, use of oral contraceptives, and history of STDs.

Racial/ ethnic differences in the prevalence of several risk factors were observed in both cases and controls. Hispanic cases and controls were more likely to be older, less educated, to report lower number of lifetime sexual partners, to be older at the time of first sexual intercourse, and to have lower rates of STDs. Meanwhile, white cases and controls were more likely to have a history of smoking, more lifetime sexual partners and African Americans were more likely to report an earlier age at sexual intercourse and to be nonsmokers.

Table 2 shows the prevalence of HPV DNA in cases and controls by racial/ ethnic group. Overall, $73 \%$ of cases and $19 \%$ of controls were HPV DNA-positive. Prevalence was higher among HSI L cases (84\%) than among LSI L cases (64\%). Racial/ethnic differences in the prevalence of HPV infection were observed in the control group ( $p=0.017$ ) but not in the case com- 
Association between HPV and SIL by racial/ethnic group and grade of lesion.

\begin{tabular}{lrrrrrr}
\hline Race/Ethnic group & \multicolumn{2}{c}{ LGSIL } & \multicolumn{2}{c}{ HGSIL } & \multicolumn{2}{c}{ SIL } \\
& COR1 & AOR2 $(95 \% \mathrm{Cl})$ & COR1 & AOR2 $(95 \% \mathrm{Cl})$ & COR1 & \multicolumn{1}{c}{ AOR2 $(95 \% \mathrm{CI})$} \\
\hline All & 7.6 & $6.6(4.1-10.4)$ & 22.0 & $24.8(13.8-44.7)$ & 11.5 & $10.8(7.1-16.4)$ \\
White & 7.5 & $7.7(3.2-18.4)$ & 26.5 & $34.6(12.8-93.8)$ & 13.5 & $15.3(6.9-33.8)$ \\
African-american & 4.2 & $4.3(1.9-9.8)$ & 8.0 & $14.2(3.6-56.0)$ & 5.8 & $5.8(2.6-12.6)$ \\
Hispanics & 11.4 & $16.6(6.5-42.3)$ & 32.6 & $78.6(23.8-260.1)$ & 17.5 & $29.5(12.4-70.5)$ \\
\hline
\end{tabular}

1 Crude OR; all COR $95 \%$ Cls exclude one.

2 OR adjusted by age, age at first sexual intercourse, number of lifetime sexual partners, history of STDs, and smoking.

bined group ( $p=0.425)$. Similarly, no racial/ ethnic differences in HPV prevalence were observed among LSIL cases $(p=0.99)$ and among HSIL cases $(p=0.59)$. In the control group, the prevalence of $\mathrm{HPV}$ infection was higher among African-American women (30\%), intermediate among white women (19\%), and lower among Hispanic women (13\%).

A strong risk of SIL (both LSIL and HSIL) was associated with HPV infection (Table 3). After adjustment for age, number of lifetime sexual partners, age at first sexual intercourse, and history of smoking and STDs the AOR for $\mathrm{SI} L$ was $10.8(95 \% \mathrm{Cl}$ 7.1-16.4). The risk was 4 times higher for $\mathrm{HSI} L(\mathrm{AOR}=24.8 ; 95 \% \mathrm{CI}$ 13.844.7) than for $\mathrm{LSIL}(\mathrm{AOR}=6.6 ; 95 \% \mathrm{Cl} 4.2-10.6$ ).

Hispanics showed a fivefold stronger association with HPV infection than African Americans and a twofold stronger risk than whites for SI L combined, and LSI L and HSIL separately. The AOR for SIL was $29.5(95 \% \mathrm{Cl} 12.4-70.5)$ for Hispanics, 5.8 (95\% Cl 2.6-12.6) for African Americans, and $15.3(95 \% \mathrm{Cl}$ 6.9-33.8) for whites. The strength of the association was three to four times higher for HSIL than LSIL. The strongest association was observed for HSI L among Hispanic women (AOR = 78.6; 95\% Cl 23.8-260.1).

\section{Discussion}

Our findings support the strong association between SI L and HPV; an association with both HSIL and LSIL but fourfold stronger for HSILs. Although this association was observed in all racial/ ethnic groups, the data suggest that significant differences in the strength of the association among groups may exist. A stronger association was observed among $\mathrm{H}$ ispanics followed by whites and African Americans.

Al though direct comparison between studies is difficult because of methodological dif- ferences, the magnitude of the association be tween HPV and SIL observed in this population, is consistent with previous studies using PCR techniques (Morrison et al., 1991; Bosch et al., 1993, 1995; Shiffman et al., 1993; Becker et al., 1994; Olsen et al., 1995; Liaw et al., 1995). Morrison et al. (1991) detected an AOR of 10.4 for the association between HPV infection and SIL (among in a inner-city multi-ethnic sample in New York, NY). Similarly, in our study we observed an AOR of 10.8 (95\% Cl 7.1-16.4) for SIL combined. These results are also consistent with studies of the association between HPV infection and grade of lesion. An increased risk for both low-grade and high-grade lesions, but stronger for high-grade lesions, have been reported. This stronger association with HSIL has been shown to be HPV-type specific. (Shiffman et al., 1993; Brisson et al., 1994; Liaw et al., 1995).

Our estimates of the association between HPV infection and high-grade cervical intraepithelail neoplasia were within range of recently reported studies using PCR-based assays (Muñoz \& Bosch, 1997). AOR ranging from 15.5 (95\% Cl 8.2-29.4) in Colombia (Bosch et al., 1993) to 122.3 (95\% Cl 38.5-388.9) in Taiwan (Liaw et al., 1995) have been reported. In Norway, Olsen et al. (1995) observed an AOR of 72.8 (95\% 27.6-191.9) and in Spain, Bosch et al (1993) reported an AOR of 56.9 (95\% Cl 24.8130.6). In the U.S., Schiffman et al. (1993) in Portland and Becker et al. (1994) in New Mexico reported an OR of $42.0(95 \% \mathrm{Cl}$ 15.3-124.3) and $20.8(95 \% \mathrm{Cl} 10.8-40.2)$, respectively. We observed risk estimates for HSIL ranging from 14.2 (95\% Cl 3.6-56.0) among African Americans to $78.6(95 \% \mathrm{Cl} 23.8-260.1)$ among Hispanics (Table 2).

The racial/ ethnic differences in HPV prevalence in the control group and therefore in the HPV-associated risk for SILs in the present study were unexpected. Particularly interesting was the low risk observed among African- 
American women for both LSILs and HSILs. $\mathrm{Hispanics}$ had a fivefold higher risk that African Americans and a twofold higher risk than whites. Similarly, white women had a 2.6 higher risk than African Americans did. Our risk estimates for HSIL among African Americans compares to that observed among Colombian women (Bosch et al., 1993), which is among the lowest risk estimates reported in recent studies using PCR techniques for HPV testing (Muñoz, 1997). There is only one published study (Becker et al., 1993) and the abstract presented at the 14th International Papillomavirus Conference in Quebec City, Canada in 1995 (Coker et al., 1995) addressing racial/ ethnic differences in HPV-associated risks for SILs in the U.S. Results from these studies are consistent with our findings. Becker et al. (1993) observed in New Mexico, a twofold higher risk among Hispanic women $(A O R=25.7 ; 95 \% \mathrm{Cl} 11.0-60.3)$ than among non-Hispanic whites (AOR $=13.3$; $95 \%$ $\mathrm{Cl}$ 5.2-34.0) using the results from the generic PCR probe. Coker et al . (1995) in South Carolina found a fourfold higher risk in whites (crude $\mathrm{OR}=21.5$ ) than in African-American women (crude OR $=5.5$ ) that persisted after controlling for other cofactors.

Despite the lack of information about racial/ethnic differences in the HPV-associated risk for cervical neoplasia, there is some data that suggest that the prevalence of HPV types may vary among different populations (Bosch et al., 1995). These differences may contribute, among other factors, to the geographic and racial/ ethnic variability in cervical cancer incidence rates. Bosch et al. (1995) observed a significant geographic variation in the prevalence of some of the less-common HPV types. HPV45 was more prevalent among cases in Western Africa and HPV-39 and HPV-59 were almost exclusively detected among Latin American cases. In support of the potential role of HPV type to explain racial/ ethnic differences in risk for cervical neoplasia, Becker et al. (1993) found the largest ethnic difference with positivity to HPV types 16/ 18 (by ViraType). HPV 16/ 18 positive Hispanic women had a 10-fold higher risk of HSIL (AOR 171.0; 95\% CI 22.8-1280.5) than non-Hispanic white women (AOR $=18.0,95 \%$ Cl 6.4-50.5).

Another possible explanation for the observed racial/ ethnic differences may be related to variability in the host immune function. The fact that only a small proportion of women with HPV infection developed cervical neoplasia and the higher risk of cervical neoplasia and progression of disease among immunosuppressed women suggest an important role of the immune system in the pathogenesis of HPV-induced disease (IARC, 1995). Host genetic factors such as human leukocyte antigens (HLA) have been also implicated in the risk of cervical neoplasias. Apple et al. (1994; 1995) found a significant association between certain HLA class II haplotypes and the risk of preinvasive and invasive cervical lesions among Hispanic women. This association was specifically observed among HPV-16 positive women. Similarly, an increased risk for cervical cancer has been associated to certain HLA class II haplotypes among Caucasian women (Wank et al., 1993) and African-American women (Gregoire et al., 1994).

It is also possible that racial/ ethnic differences in other risk factors such as socioeconomic characteristics, sexual behaviors, smoking habits, reproductive and dietary factors, history of STDs, and in other cofactors not measured in the present study (characteristics of male partners and endogenous hormonal factors) might explain our results. For example, socioeconomic factors may operate differently among different racial/ethnic groups. Level of education has been found to be an independent factor for cervical neoplasia. Peters et al. (1986) found years of education inversely associated with risk of cervical cancer among Hispanic women in Los Angeles County. Similarly, Becker et al. (1993) observed a higher risk of HSIL among women with lower educational attainment. In our study statistically significant racial/ ethnic differences in educational levels in both cases and controls were observed; however, we did not find educational level to be an independent risk factor for SI L among racial/ ethnic combined or among each group separately (data not shown).

There are some potential limitations in the present study that need to be considered in the interpretation of these findings. First, the study design (clinic-based case-control study) limits the generalization of the results. However, in case-control studies the goal is to select a group of controls that represent the population source of the cases and not the entire population of nondisease individuals (Rothman \& Greenland, 1998). Cases were selected at one of the largest colposcopy clinics in Harris County providing services to low-income and minority women from city and county Health Department Clinics. Controls were identified at two HCHD clinics with a large multi-ethnic based population that referred women with abnormal cytology to the UTMDACC colposcopy clinic. Cases and controls were ascertained among all eligible women and prior to knowl- 
edge of final cytological or histological diagnosis and HPV status. In addition, data collection instruments were translated to Spanish and bilingual interviewers were available in order to insure participation among non-English speaking Hispanics. The prevalence of known risk factors for cervical neoplasia in both cases and controls was consistent with previous studies. Most factors (such as number of sexual partners, age at first intercourse, smoking, and history of STDs) were found to be significantly associated with the risk of SIL in univariate logistic models (data not shown).

Another potential factor affecting the interpretation of the results is the unbalanced racial/ ethnic distribution of cases and controls, particularly for white women (Table 1). As mentioned in the methods section, this imbalance was expected given the racial/ ethnic distribution of the patient population at the UTMDACC colposcopy clinic and the study recruitment procedures. We had initially planned to select a random sample of white cases to obtain a case:control ratio of one. In a preliminary analysis we estimated crude OR for the association between SIL and HPV for the total sample (including all white) and for a subsample including only a random sample of the available white cases. Similar crude risk estimates were obtained with the total sample (OR $=10.0,95 \% \mathrm{Cl} 6.7-15.1$ ) and the subsample (OR $=9.9 ; 95 \% \mathrm{Cl}$ 4.5-21.8). Therefore, the unbalanced number of cases and controls among white women does not seem to have biased the estimate of risk. Furthermore, the prevalence of risk factors (education, smoking history, number of sexual partners, age at first intercourse and history of STDs) in both cases and controls was consistent with national data.

Although the possibility of interviewer, recall, and information bias can not be eliminated, attempts were made to minimize them using a structured computer-assisted interview, training and supervising interviewers, conducting data collection both in English and Spanish and prior to knowledge of the final diagnosis and HPV status, and by reminding women about the confidentiality of the information and the importance of the accuracy of their responses.

Misclassification of HPV and disease status needs to be also consi dered in the interpretation. The cross-sectional nature of the HPV testing may not accurately reflect the true prevalence in the study population. Despite this limitation the preval ence of HPV observed in the current study, in both cases and controls, is consistent with previous studies using PCR methods (Muñoz, 1997). Similarly, the racial/ ethnic differences in the prevalence of HPV infection in the control group is consistent with the data reported by Hildesheim et al. (1993). These authors also found a higher prevalence of HPV infection among African Americans (43.9\%), intermediate in whites $(32.1 \%)$, and lower in Hispanics (25.5\%) among a clinicbased population of women with normal cytologic smear residing in the Washington, DC area (Hildesheim et al., 1993).

The difference in the ascertainment of disease status in cases (at the Department of Pathology UTMDACC) and controls (at a reference laboratory) may have introduced misclassification particularly among the control group. Our decision for this difference in ascertainment procedures was based on results from a small pilot study $(n=100)$ by one of the authors in this study (MFM) to compare the agreement between the reading at the reference laboratory and the Department of Pathology. A high agreement was observed between the readers, kappa coefficient of 0.85 (personal communication). Furthermore, most women in the control group (98\%) had a negative cytology at the time of entry, all had a negative history of cervical abnormalities, 93\% have had a Pap smear within the last year prior to enrollment (85\% reported having annual Pap smears), and based on our preliminary analysis of HPV type 36 of the 53 HPV positive controls were positive for non high-risk HPV types. Based on these characteristics the likelihood of fal se negative results among the controls should be low.

Despite these limitations, we have shown a strong association between HPV infection and SIL with stronger association for HSIL in this multi-ethnic population in Harris County, Texas. We have also shown differences in the strength of the association among racial/ ethnic group. The pattern of risk among the racial ethnic groups appears to be consist with the limited information available addressing this issue. Further research is needed to confirm these findings, and to understand the factors influencing these racial/ eth nic differences as well as their implications for prevention and control of cervical neoplasia. 
References

APPLE, R. J.; ERLICH, H. A.; KLITZ, W.; MANOS, M . M.; BECKER, T. M. \& WHEELER, C. M., 1994. HLA DR-DQ associations with cervical carcinoma show papillomavirus-type specificity. Nature Genetics, 6:157-162.

APPLE, R. J.; BECKER, T. M.; WHEELER, C. M. \& ER$\mathrm{LICH}, \mathrm{H}$. A., 1995. Comparison of human leukocyte antigen DR-DQ disease associations found with cervical dysplasia and invasive cervical carcinoma. Journal of the National Cancer Institute, 87: 427-436.

BECKER, T. M.; WHEELER, C. M.; KEY, C. R. \& SAMET, J. M., 1992. Cervical cancer incidence and mortality in New Mexico's Hispanic, American Indians, and non-Hispanic whites. Western Journal of Medicine, 156:376-379.

BECKER, T. M.; WHEELER, C. M.; MCGOUGH, S.; PARMENTER, C. A.; JORDAN, S. W.; STIDLEY, C. A.; MCPHERSON, R. S. \& DORIN, M. H., 1994. Sexually transmitted diseases and other risk factors for cervical dysplasia among Southwestern Hispanic and non-Hispanic white women. JAMA, 271:1181-1188.

BLOCK, G., 1989. Health Habits and History Questionnaire: Diet, History and Other Risk Factors. Personal Computer System Packet. Washington, D. C.: Division of Cancer Prevention and Control, $\mathrm{Na}-$ tional Cancer Institute/ National Institutes of Health.

BOSCH, F. X.; MUÑOZ, N.; DE SANJOSE, S.; NAVARRO, C.; MOREO, P.; ASCUNCE, N.; GONZALEZ, L. C.; TAFUR, L.; GILI, M.; LARRAÑAGA, I.; VILADIU, P.; DANIEL, R. W.; ALONZO DE RUIZ, P.; ARISTIZABAL, N.; SANTAMARIA, M.; GUERRERO, E. \& SHAH, K., 1993. Human papillomavirus and cervical intraepithelial neoplasia grade III/Carcinoma in situ: a case-control study in Spain and Colombia. Cancer Epidemiology, Biomarkers and Prevention, 2:415-422.

BOSCH, F. X.; MANOS, M. M.; MUÑOZ, N.; SHERMAN, M.; JANSEN, A. M.; PETO, J.; SCHIFFMAN, M. H.; MORENO, V.; KURMAN, R. \& SHAH, K. V, 1995. Prevalence of human papillomavirus in cervical cancer: a worldwide perspective. International Biological Study on Cervical Cancer (IBSCC). Journal of the National Cancer Institute, 87:796-802.

BRISSON, J.; MORIN, C.; FORTIER, M.; ROY, M.; BOUCHARD, C.; LECLERC, J.; CHRISTEN, A.; GUIMONT, C.; PENAULT, F. \& MEISELS, A., 1994. Risk factors for cervical intrepithelial neoplasia: differences between low-and high-grade lesions. American Journal of Epidemiology, 140:700-710.

COKER, A. L.; KNIGHT, S. M.; JENKINS, G. R. \& PIRISI, L., 1995. Racial differences in risk factors for cervical neoplasia. 14th International Papillomavirus Conference, Abstracts. Quebec: 14th International Papillomavirus Conference.

FÖRSTER, V. T., 1948. Zwischenmolekulare energiewanderung und fluoreszenz. Annals of Physics, 2:55-75.

GREGOIRE, L.; LAWRENCE, W. D.; KUKURUGA, D.; EISENBREY, A. B. \& LANCANTER, W. D., 1994. Association between HLA-DQB1 alleles and the risk of cervical cancer in African-American women. International Journal of Cancer, 57:504-507.

HILDESHEIM, A.; GRAVITT, P.; SCHIFFMAN, M. K.; KURMAN, R. J.; BARNES, W.; JONES, S.; TCHABO, J. G.; BRINTON, L. A.; COPELAND, C.; EPP, J. \& MANOS, M. M., 1993. Determinants of genital human papillomavirus infection in low-income women in Washington, D. C. Sexually Transmitted Diseases, 20:279-285.

HOSMER, D. W. \& LEMESHOW, S., 1989. Applied Logistic Regression. New York: John Wiley \& Sons.

IARC WORKING GROUP, 1995. Human Papillomaviruses. IARC Monographs on the Evaluation of Carcinogenic Risk to Human, vol. 64. Lyon: International Agency for Research on Cancer.

LIAW, K. L.; HSING, A. W.; CHEN, C. J.; SCHIFFMAN, M. H.; ZHANG, T. Y.; HSIEH, C. Y.; GREER, C. E.; YOU, S. L.; HUANG, T. W.; WU, T. C.; O'LEARY, T. J.; SEIDMAN, J. D.; BLOT, W. J.; MEINERT, C. L. \& MANOS, M. M., 1995. Human papilloma virus and cervical neoplasia: a case-control study in Tai wan. International Journal of Cancer, 62:565571.

MANOS, M. M.; TING, Y.; WRIGHT, D. K.; LEWIS, A. J .; BROKER, T. R. \& WOLINSKY, S. M., 1989. Use of polymerase chain reaction amplification for the detection of genital human papillomaviruses. Cancer Cells, 7:209-214.

MILLER, B. A.; KOLONEL, L. N.; BERSTEIN, L.; YOUNG J., J. L.; SWANSON, G. M.; KEY, C. R.; LIFF, J. M.; GLOVER, C. S. \& ALEXANDER, G. A., 1996. Racial/Ethnic Patterns of Cancer in the United States 1988-1992. Bethesda: National Cancer Institute. NIH Pub. N 96-4104.

MORRISON, E. A. B.; HO, G. Y. F.; VERMUND, S. H.; GOLBERG, G. L.; KADISH, A. S.; KELLEY, K. F. \& BURK, D. B., 1991. Human papillomavirus infection and other risk factors for cervical neoplasia: a case-control study. International Journal of Cancer, 49:6-13.

MUÑOZ, N., 1997. Human papillomavirus and cervical cancer. In: New Developments in Cervical Cancer Screening and Prevention (E. Franco \&J. Monsonego, eds.), pp. 3-13. Cambridge: Blackwell Science.

MUÑOZ, N. \& BOSCH, F. X., 1997. Cervical cancer and human papillomavirus: epidemiological evidence and perspective for prevention. Salud Pública de México, 39:274-282.

NAPOLES-SPRINGER, A.; PEREZ-STABLE, E. J. \& WASHINGTON, E., 1996. Risk factors for invasive cervical cancer in Latino women. Journal of Medical Systems, 20:277-293.

OLSEN, A. O.; GJ OEN, K.; SAUER, T.; ORSTAVIK, I.; NAESS, O.; KIERULF, K.; SPONLAND, G. \& MAGNUS, P., 1995. Human papillomavirus and cervical intraepithelial neoplasia grade II-III : a population-based case-control study. International Journal of Cancer, 61:312-315.

PETERS, R. K.; THOMAS, D.; HAGAN, D.; THOMAS, M. \& HENDERSON, B., 1986. Risk factors for invasive cervical cancer among latinas and nonlatinas in Los Angeles County. Journal of the National Cancer Institute, 77:1063-1077. 
ROTHMAN, K. J. \& GRENLAND, S., 1998. Case-control studies. In: Modern Epidemiology (K. J. Rothman \& S. Grenland, eds.), pp. 93-114, Philadelphia: Lippincott-Raven.

SCHIFFMAN, M. H.; BAUER, H. M.; HOOVER, R. N.; GLASS, A. G., CADELL, D. M.; RUSH, B. B.; SCOTT, D. R.; SHERMAN, M. E.; KURMAN, R. J.; WACHOLDER, S.; STATON, C. K. \& MANOS, M. M., 1993. Epidemi ology evidence showing that human papillomavirus infection causes most cervical intraepithelial neoplasia. Journal of the $\mathrm{Na}$ tional Cancer Institute, 85:958-964.

SPSS, 1997. Statistical Package for the Social Sciences for Windows: Version 8.0.0. Chapel Hill: SPSS.
STATACORP., 1997. Stata Stati stical Software: Release 5.0. College Station: Stata Corporation.

SWAN, D. C.; TUCKER, R. A.; HOLLOWAY, B. P. \&. ICEN OGLE, J. P, 1997. A sensitive, type-specific, fluorogenic probe assay for detection of human papillomavirus DNA. Journal of Clinical Microbiology, 35:886-891.

WANK, R.; TER MEULEN, J.; LUANDE, J.; EBERHARDT, H. C. \& PAWLITA, M., 1993. Cervical intraepithelial neoplasia, cervical carcinoma, and risk for patients with HLA-DQB $1 * 0602, * 0301$, *0303 alleles. Lancet, 341:1215-1219. 